\title{
Homoclinic orbits for second-order discrete Hamiltonian systems with subquadratic potential
}

\section{Xiaoyan Lin*}

\section{"Correspondence:}

xiaoyanlin98@hotmail.com Department of Mathematics, Huaihua College, Huaihua, Hunan 418008 , P.R. China

\begin{abstract}
Under the assumptions that $W(n, x)$ is indefinite sign and subquadratic as $|x| \rightarrow+\infty$ and $L(n)$ satisfies

$$
\liminf _{|n| \rightarrow+\infty}\left[|n|^{\nu-2} \inf _{|x|=1}(L(n) x, x)\right]>0
$$

for some constant $v<2$, we establish a theorem on the existence of infinitely many homoclinic solutions for the second-order self-adjoint discrete Hamiltonian system

$$
\Delta[p(n) \Delta u(n-1)]-L(n) u(n)+\nabla W(n, u(n))=0,
$$

where $p(n)$ and $L(n)$ are $\mathcal{N} \times \mathcal{N}$ real symmetric matrices for all $n \in \mathbb{Z}$, and $p(n)$ is always positive definite.

MSC: 39A11; 58E05; 70H05

Keywords: homoclinic solution; discrete Hamiltonian system; subquadratic; critical point
\end{abstract}

\section{Introduction}

Consider the second-order self-adjoint discrete Hamiltonian system

$$
\triangle[p(n) \triangle u(n-1)]-L(n) u(n)+\nabla W(n, u(n))=0,
$$

where $n \in \mathbb{Z}, u \in \mathbb{R}^{\mathcal{N}}, \Delta u(n)=u(n+1)-u(n)$ is the forward difference, $p, L: \mathbb{Z} \rightarrow \mathbb{R}^{\mathcal{N} \times \mathcal{N}}$ and $W: \mathbb{Z} \times \mathbb{R}^{\mathcal{N}} \rightarrow \mathbb{R}, W(n, x)$ is continuously differentiable in $x$ for every $n \in \mathbb{Z}$.

As usual, we say that a solution $u(n)$ of system (1.1) is homoclinic (to 0$)$ if $u(n) \rightarrow 0$ as $n \rightarrow \pm \infty$. In addition, if $u(n) \not \equiv 0$ then $u(n)$ is called a nontrivial homoclinic solution.

The existence and multiplicity of nontrivial homoclinic solutions for problem (1.1) have been extensively investigated in the literature with the aid of critical point theory and variational methods; see, for example, [1-13]. Most of them treat the case where $W(n, x)$ is superquadratic as $|x| \rightarrow \infty$.

Compared to the superquadratic case, as far as the author is aware, there are a few papers $[10,12,13]$ concerning the case where $W(n, x)$ has subquadratic growth at infinity. 
Specifically, [12] and [10] dealt with the existence and multiplicity of homoclinic solutions for (1.1) under the following assumptions on $L$ :

$\left(\mathrm{L}_{*}\right) L(n)$ is an $\mathcal{N} \times \mathcal{N}$ real symmetric positive definite matrix for all $n \in \mathbb{Z}$ and there exists a constant $\beta>0$ such that

$$
(L(n) x, x) \geq \beta|x|^{2}, \quad \forall(n, x) \in \mathbb{Z} \times \mathbb{R}^{\mathcal{N}}
$$

$\left(\mathrm{L}_{v}\right) L(n)$ is an $\mathcal{N} \times \mathcal{N}$ real symmetric positive definite matrix for all $n \in \mathbb{Z}$ and there exists a constant $v<2$ such that

$$
\liminf _{|n| \rightarrow+\infty}\left[|n|^{\nu-2} \inf _{|x|=1}(L(n) x, x)\right]>0,
$$

respectively. In the above two cases, since $L(n)$ is positive definite, the variational functional associated with system (1.1) is bounded from below, techniques based on the genus properties have been well applied. In particular, Clark's theorem is an efficacious tool to prove the existence and multiplicity of homoclinic solutions for system (1.1). However, if $L(n)$ is not global positive definite on $\mathbb{Z}$, the problem is far more difficult as 0 is a saddle point rather than a local minimum of the variational functional, which is strongly indefinite and it is not easy to obtain the boundedness of the Palais-Smale sequence. In a recent paper [13], based on a new direct sum decomposition of the 'work space, Tang and Lin proved the following theorem by using a linking theorem which was developed in [14].

Theorem 1.1 [13] Assume that $p(n)$ is an $\mathcal{N} \times \mathcal{N}$ real symmetric positive definite matrix for all $n \in \mathbb{Z}, L$ and $W$ satisfy the following assumptions:

$\left(\mathrm{L}_{v}^{\prime}\right) \quad L(n)$ is an $\mathcal{N} \times \mathcal{N}$ real symmetric matrix for all $n \in \mathbb{Z}$ and there exists a constant $v<2$ such that

$$
\liminf _{|n| \rightarrow+\infty}\left[|n|^{v-2} \inf _{|x|=1}(L(n) x, x)\right]>0 ;
$$

(W1) there exist constants $\max \{1,2 /(3-v)\}<\gamma_{1}<\gamma_{2}<2$ and $a_{1}, a_{2} \geq 0$ such that

$$
|W(n, x)| \leq a_{1}|x|^{\gamma_{1}}+a_{2}|x|^{\gamma_{2}}, \quad \forall(n, x) \in \mathbb{Z} \times \mathbb{R}^{\mathcal{N}}
$$

(W2) there exists a function $\varphi \in C([0,+\infty),[0,+\infty))$ such that

$$
|\nabla W(n, x)| \leq \varphi(|x|), \quad \forall(n, x) \in \mathbb{Z} \times \mathbb{R}^{\mathcal{N}}
$$

where $\varphi(s)=O\left(s^{\gamma_{3}-1}\right)$ as $s \rightarrow 0^{+}, \max \{1,2 /(3-v)\}<\gamma_{3}<2$;

(W3) there exist constants $b_{1}>0, b_{2}, b_{3} \geq 0$ and $\max \{1,2 /(3-v)\}<\gamma_{6}<\gamma_{5}<\gamma_{4}<2$ such that

$$
2 W(n, x)-\nabla W(n, x) \geq b_{1}|x|^{\gamma_{4}}-b_{2}|x|^{\gamma_{5}}-b_{3}|x|^{\gamma_{6}}, \quad \forall(n, x) \in \mathbb{Z} \times \mathbb{R}^{N}
$$

(W4) there exist constants $b_{4}>0, b_{5}, b_{6} \geq 0$ and $\max \{1,2 /(3-v)\}<\gamma_{7}<\gamma_{8}<\gamma_{9}<2$ such that

$$
W(n, x) \geq b_{4}|x|^{\gamma_{7}}-b_{5}|x|^{\gamma_{8}}-b_{6}|x|^{\gamma_{9}}, \quad \forall(n, x) \in \mathbb{Z} \times \mathbb{R}^{\mathcal{N}} ;
$$


(W5) $W(n,-x)=W(n, x), \forall(n, x) \in \mathbb{Z} \times \mathbb{R}^{\mathcal{N}}$.

Then system (1.1) possesses infinitely many nontrivial homoclinic solutions.

We remark that the condition 'positive definite' is removed in $\left(\mathrm{L}_{v}^{\prime}\right)$, i.e., $L(n)$ is not required to be global positive definite on $\mathbb{Z}$. The main goal of this paper is to weaken conditions (W1), (W2), (W3) and (W4) of Theorem 1.1 under assumption $\left(\mathrm{L}_{v}^{\prime}\right)$.

To state our result, we first introduce the following assumptions:

(W1') there exist constants $\sigma_{i} \in[0,2-v), a_{i} \geq 0$ and $\max \left\{1,2\left(1+\sigma_{i}\right) /(3-v)\right\}<\gamma_{i}<2$ with $i=1,2$ such that

$$
|W(n, x)| \leq \sum_{i=1}^{2} a_{i}\left(1+|n|^{\sigma_{i}}\right)|x|^{\gamma_{i}}, \quad \forall(n, x) \in \mathbb{Z} \times \mathbb{R}^{\mathcal{N}} ;
$$

(W2') there exist two constants $\max \left\{1,2\left(1+\sigma_{i}\right) /(3-v)\right\}<\gamma_{i+2}<2, i=1,2$ and two functions $\varphi_{1}, \varphi_{2} \in C([0,+\infty),[0,+\infty))$ such that

$$
|\nabla W(n, x)| \leq \sum_{i=1}^{2}\left(1+|n|^{\sigma_{i}}\right) \varphi_{i}(|x|), \quad \forall(n, x) \in \mathbb{Z} \times \mathbb{R}^{\mathcal{N}},
$$

where $\varphi_{i}(s)=O\left(s^{\gamma_{i+2}-1}\right)$ as $s \rightarrow 0^{+}, i=1,2$;

(W3') there exist constants $b_{1}>0, b_{2} \geq 0$ and $1<\gamma_{6}<\gamma_{5}<2$ such that

$$
2 W(n, x)-\nabla W(n, x) \geq b_{1}|x|^{\gamma_{5}}-b_{2}|x|^{\gamma_{6}}, \quad \forall(n, x) \in \mathbb{Z} \times \mathbb{R}^{N}
$$

(W4') there exist constants $b_{3}>0, b_{4} \geq 0$ and $1<\gamma_{7}<\gamma_{8}<2$ such that

$$
W(n, x) \geq b_{3}|x|^{\gamma_{7}}-b_{4}|x|^{\gamma_{8}}, \quad \forall(n, x) \in \mathbb{Z} \times \mathbb{R}^{\mathcal{N}} .
$$

We are now in a position to state the main result of this paper.

Theorem 1.2 Assume that $p(n)$ is an $\mathcal{N} \times \mathcal{N}$ real symmetric positive definite matrix for all $n \in \mathbb{Z}, L$ and $W$ satisfy $\left(\mathrm{L}_{v}^{\prime}\right),\left(\mathrm{W} 1^{\prime}\right),\left(\mathrm{W} 2^{\prime}\right),\left(\mathrm{W} 3^{\prime}\right),\left(\mathrm{W} 4^{\prime}\right)$ and (W5). Then system (1.1) possesses infinitely many nontrivial homoclinic solutions.

\section{Preliminaries}

In what follows, we always assume that $p(n)$ is a real symmetric positive definite matrix for all $n \in \mathbb{Z}$. As done in [13], we define

$$
l(n)=\inf _{x \in \mathbb{R}^{\mathcal{N}},|x|=1}(L(n) x, x)
$$

and

$$
\mathbb{Z}^{1}=\{n \in \mathbb{Z}: l(n) \leq 0\}, \quad \mathbb{Z}^{2}=\{n \in \mathbb{Z}: l(n)>0\} .
$$

Then by $\left(\mathrm{L}_{v}^{\prime}\right), l(n)$ is bounded from below and so $\mathbb{Z}^{1}$ is a finite set and

$$
l_{*}:=\min \left\{l(n): n \in \mathbb{Z}^{2}\right\}>0 .
$$


Define

$$
\tilde{L}(n)=\left\{\begin{array}{ll}
l_{*} I_{\mathcal{N}}, & n \in \mathbb{Z}^{1}, \\
L(n), & n \in \mathbb{Z}^{2} ;
\end{array} \quad \tilde{l}(n)= \begin{cases}l_{*}, & n \in \mathbb{Z}^{1} \\
l(n), & n \in \mathbb{Z}^{2}\end{cases}\right.
$$

Then, it follows from (2.1), (2.2), (2.3) and (2.4) that

$$
(\tilde{L}(n) x, x) \geq \tilde{l}(n)|x|^{2} \geq l_{*}|x|^{2}, \quad \forall(n, x) \in \mathbb{Z} \times \mathbb{R}^{\mathcal{N}}
$$

Let

$$
\begin{aligned}
& S=\left\{\{u(n)\}_{n \in \mathbb{Z}}: u(n) \in \mathbb{R}^{\mathcal{N}}, n \in \mathbb{Z}\right\} \\
& E=\left\{u \in S: \sum_{n \in \mathbb{Z}}[(p(n+1) \Delta u(n), \Delta u(n))+(\tilde{L}(n) u(n), u(n))]<+\infty\right\}
\end{aligned}
$$

and for $u, v \in E$, let

$$
(u, v)=\sum_{n \in \mathbb{Z}}[(p(n+1) \Delta u(n), \Delta v(n))+(\tilde{L}(n) u(n), v(n))] .
$$

Then $E$ is a Hilbert space with the above inner product, and the corresponding norm is

$$
\|u\|=\left\{\sum_{n \in \mathbb{Z}}[(p(n+1) \Delta u(n), \Delta u(n))+(\tilde{L}(n) u(n), u(n))]\right\}^{1 / 2}, \quad u \in E
$$

As usual, for $1 \leq q<+\infty$, set

$$
l^{q}\left(\mathbb{Z}, \mathbb{R}^{\mathcal{N}}\right)=\left\{\{u(n)\}_{n \in \mathbb{Z}}: u(n) \in \mathbb{R}^{\mathcal{N}}, n \in \mathbb{Z}, \sum_{n \in \mathbb{Z}}|u(n)|^{q}<+\infty\right\}
$$

and

$$
l^{\infty}\left(\mathbb{Z}, \mathbb{R}^{\mathcal{N}}\right)=\left\{\{u(n)\}_{n \in \mathbb{Z}}: u(n) \in \mathbb{R}^{\mathcal{N}}, n \in \mathbb{Z}, \sup _{n \in \mathbb{Z}}|u(n)|<+\infty\right\}
$$

and their norms are defined by

$$
\begin{aligned}
& \|u\|_{q}=\left(\sum_{n \in \mathbb{Z}}|u(n)|^{q}\right)^{1 / q}, \quad \forall u \in l^{q}\left(\mathbb{Z}, \mathbb{R}^{\mathcal{N}}\right) ; \\
& \|u\|_{\infty}=\sup _{n \in \mathbb{Z}}|u(n)|, \quad \forall u \in l^{\infty}\left(\mathbb{Z}, \mathbb{R}^{\mathcal{N}}\right),
\end{aligned}
$$

respectively.

Lemma 2.1 [9, Lemma 2.2] For $u \in E$, one has

$$
\|u\|_{\infty} \leq \frac{1}{\sqrt[4]{\left(l_{*}+4 \alpha\right) l_{*}}}\|u\|
$$

where $\alpha=\inf \left\{(p(n) x, x): n \in \mathbb{Z}, x \in \mathbb{R}^{\mathcal{N}},|x|=1\right\}$. 
Set

$$
b(u, v)=\sum_{n \in \mathbb{Z}}[(p(n+1) \Delta u(n), \Delta v(n))+(L(n) u(n), v(n))], \quad \forall u, v \in E .
$$

Lemma 2.2 [13, Lemma 2.3] Suppose that L satisfies $\left(\mathrm{L}_{v}^{\prime}\right)$. Then

(i) $b(u, v)$ is a bilinear function on $E$, and there exists a constant $C_{0}>0$ such that

$$
|b(u, v)| \leq C_{0}\|u\|\|v\|, \quad \forall u, v \in E
$$

(ii)

$$
b(u, u)=\|u\|^{2}-\sum_{n \in \mathbb{Z}^{1}}((\tilde{L}(n)-L(n)) u(n), u(n)), \quad \forall u \in E .
$$

By $\left(\mathrm{L}_{v}^{\prime}\right)$, there exist an integer $N_{0}>\max \left\{|n|: n \in \mathbb{Z}^{1}\right\}$ and $M_{0}>0$ such that

$$
|n|^{v-2} \inf _{|x|=1}(L(n) x, x) \geq M_{0}, \quad|n| \geq N_{0}
$$

which implies

$$
|n|^{\nu-2}(L(n) x, x) \geq M_{0}|x|^{2}, \quad|n| \geq N_{0}, x \in \mathbb{R}^{\mathcal{N}}
$$

Lemma 2.3 Suppose that L satisfies $\left(\mathrm{L}_{v}^{\prime}\right)$. Then, for $\sigma \in[0,2-v)$ and $1 \leq q \in(2(1+\sigma) /(3-$ v), 2), E is compactly embedded in $l^{q}\left(\mathbb{Z}, \mathbb{R}^{\mathcal{N}}\right)$; moreover,

$$
\sum_{|n|>N}\left(1+|n|^{\sigma}\right)|u(n)|^{q} \leq \frac{K(\sigma, q)}{N^{\kappa}}\|u\|^{q}, \quad \forall u \in E, N \geq N_{0}
$$

and

$$
\begin{aligned}
& \sum_{n \in \mathbb{Z}}\left(1+|n|^{\sigma}\right)|u(n)|^{q} \leq\left[\left(\sum_{|n| \leq N}\left(1+|n|^{\sigma}\right)^{2 /(2-q)}[\tilde{l}(n)]^{-q /(2-q)}\right)^{1-\frac{q}{2}}+\frac{K(\sigma, q)}{N^{\kappa}}\right]\|u\|^{q}, \\
& \forall u \in E, N \geq N_{0},
\end{aligned}
$$

where

$$
\kappa=\frac{(3-v) q-2(1+\sigma)}{2}>0, \quad K(\sigma, q)=2\left[\frac{2(2-q)}{(3-v) q-2(1+\sigma)}\right]^{1-\frac{q}{2}} M_{0}^{-q / 2}
$$

Proof Let $r=[(3-v) q-2(1+\sigma)] /(2-q)$. Then $r>0$. For $u \in E$ and $N \geq N_{0}$, it follows from (2.10), (2.13) and the Hölder inequality that

$$
\begin{aligned}
\sum_{|n|>N}\left(1+|n|^{\sigma}\right)|u(n)|^{q} & \leq 2\left(\sum_{|n|>N}|n|^{-[(2-v) q-2 \sigma] /(2-q)}\right)^{1-\frac{q}{2}}\left(\sum_{|n|>N}|n|^{2-v}|u(n)|^{2}\right)^{\frac{q}{2}} \\
& =2\left(\sum_{|n|>N}|n|^{-(r+1)}\right)^{1-\frac{q}{2}}\left(\sum_{|n|>N}|n|^{2-v}|u(n)|^{2}\right)^{\frac{q}{2}}
\end{aligned}
$$




$$
\begin{aligned}
& \leq 2\left(\frac{2}{r N^{r}}\right)^{1-\frac{q}{2}}\left[\frac{1}{M_{0}} \sum_{|n|>N}(L(n) u(n), u(n))\right]^{\frac{q}{2}} \\
& \leq \frac{2^{1+(2-q) / 2}}{M_{0}^{q / 2} r^{(2-q) / 2} N^{\kappa}}\|u\|^{q} \\
& =\frac{K(\sigma, q)}{N^{\kappa}}\|u\|^{q} .
\end{aligned}
$$

This shows that (2.11) holds. Hence, from (2.5), (2.11) and the Hölder inequality, one has

$$
\begin{aligned}
& \sum_{n \in \mathbb{Z}}\left(1+|n|^{\sigma}\right)|u(n)|^{q} \\
& \quad=\sum_{|n| \leq N}\left(1+|n|^{\sigma}\right)|u(n)|^{q}+\sum_{|n|>N}\left(1+|n|^{\sigma}\right)|u(n)|^{q} \\
& \quad \leq\left(\sum_{|n| \leq N}\left(1+|n|^{\sigma}\right)^{2 /(2-q)}[\tilde{l}(n)]^{-q /(2-q)}\right)^{1-\frac{q}{2}}\left(\sum_{|n| \leq N} \tilde{l}(n)|u(n)|^{2}\right)^{\frac{q}{2}}+\frac{K(\sigma, q)}{N^{\kappa}}\|u\|^{q} \\
& \quad \leq\left(\sum_{|n| \leq N}\left(1+|n|^{\sigma}\right)^{2 /(2-q)}[\tilde{l}(n)]^{-q /(2-q)}\right)^{1-\frac{q}{2}}\|u\|^{q}+\frac{K(\sigma, q)}{N^{\kappa}}\|u\|^{q} .
\end{aligned}
$$

This shows that (2.12) holds.

Finally, we prove that $E$ is compactly embedded in $l^{q}\left(\mathbb{Z}, \mathbb{R}^{\mathcal{N}}\right)$. Let $\left\{u_{k}\right\} \subset E$ be a bounded sequence. Then by (2.6), there exists a constant $\Lambda>0$ such that

$$
\left\|u_{k}\right\|_{\infty} \leq \frac{1}{\sqrt[4]{\left(l^{*}+4 \alpha\right) l^{*}}}\left\|u_{k}\right\| \leq \Lambda, \quad k \in \mathbb{N}
$$

Since $E$ is reflexive, $\left\{u_{k}\right\}$ possesses a weakly convergent subsequence in $E$. Passing to a subsequence if necessary, it can be assumed that $u_{k} \rightarrow u_{0}$ in $E$. It is easy to verify that

$$
\lim _{k \rightarrow \infty} u_{k}(n)=u_{0}(n), \quad \forall n \in \mathbb{Z}
$$

For any given number $\varepsilon>0$, we can choose $N_{\varepsilon}>0$ such that

$$
\frac{2^{q-1} K(\sigma, q)}{N_{\varepsilon}^{\kappa}}\left\{\left[\sqrt[4]{\left(l^{*}+4 \alpha\right) l^{*}} \Lambda\right]^{q}+\left\|u_{0}\right\|^{q}\right\}<\varepsilon
$$

It follows from (2.15) that there exists $k_{0} \in \mathbb{N}$ such that

$$
\sum_{|n| \leq N_{\varepsilon}}\left|u_{k}(n)-u_{0}(n)\right|^{q}<\varepsilon \quad \text { for } k \geq k_{0} .
$$

On the other hand, it follows from (2.11), (2.14) and (2.16) that

$$
\begin{aligned}
\sum_{|n|>N_{\varepsilon}}\left|u_{k}(n)-u_{0}(n)\right|^{q} & \leq 2^{q-1} \sum_{|n|>N_{\varepsilon}}\left(\left|u_{k}(n)\right|^{q}+\left|u_{0}(n)\right|^{q}\right) \\
& \leq \frac{2^{q-1} K(\sigma, q)}{N_{\varepsilon}^{\kappa}}\left(\left\|u_{k}\right\|^{q}+\left\|u_{0}\right\|^{q}\right)
\end{aligned}
$$




$$
\begin{aligned}
& \leq \frac{2^{q-1} K(\sigma, q)}{N_{\varepsilon}^{\kappa}}\left\{\left[\sqrt[4]{\left(l^{*}+4 \alpha\right) l^{*}} \Lambda\right]^{q}+\left\|u_{0}\right\|^{q}\right\} \\
& \leq \varepsilon, \quad k \in \mathbb{N} .
\end{aligned}
$$

Since $\varepsilon$ is arbitrary, combining (2.17) with (2.18), we get

$$
\left\|u_{k}-u_{0}\right\|_{q}^{q}=\sum_{n \in \mathbb{Z}}\left|u_{k}(n)-u_{0}(n)\right|^{q} \rightarrow 0 \quad \text { as } k \rightarrow \infty .
$$

This shows that $\left\{u_{k}\right\}$ possesses a convergent subsequence in $l^{q}\left(\mathbb{Z}, \mathbb{R}^{\mathcal{N}}\right)$. Therefore, $E$ is compactly embedded in $l^{q}\left(\mathbb{Z}, \mathbb{R}^{\mathcal{N}}\right)$ for $1 \leq q \in(2(1+\sigma) /(3-v), 2)$.

Lemma 2.4 Suppose that $L$ and $W$ satisfy $\left(\mathrm{L}_{v}^{\prime}\right)$ and (W1'). Then, for $u \in E$,

$$
\sum_{n \in \mathbb{Z}}|W(n, u(n))| \leq \phi_{1}(N)\|u\|^{\gamma_{1}}+\phi_{2}(N)\|u\|^{\gamma_{2}}, \quad N \geq N_{0},
$$

where

$$
\begin{aligned}
& \kappa_{1}=\frac{(3-v) \gamma_{1}-2\left(1+\sigma_{1}\right)}{2}, \quad \kappa_{2}=\frac{(3-v) \gamma_{2}-2\left(1+\sigma_{2}\right)}{2} ; \\
& \phi_{1}(N)=a_{1}\left[\left(\sum_{|n| \leq N}\left(1+|n|^{\sigma_{1}}\right)^{2 /\left(2-\gamma_{1}\right)}[\tilde{l}(n)]^{-\gamma_{1} /\left(2-\gamma_{1}\right)}\right)^{1-\frac{\gamma_{1}}{2}}+\frac{K\left(\sigma_{1}, \gamma_{1}\right)}{N^{\kappa_{1}}}\right] \\
& \phi_{2}(N)=a_{2}\left[\left(\sum_{|n| \leq N}\left(1+|n|^{\sigma_{2}}\right)^{2 /\left(2-\gamma_{2}\right)}[\tilde{l}(n)]^{-\gamma_{2} /\left(2-\gamma_{2}\right)}\right)^{1-\frac{\gamma_{2}}{2}}+\frac{K\left(\sigma_{2}, \gamma_{2}\right)}{N^{\kappa_{2}}}\right] .
\end{aligned}
$$

Proof For $N \geq N_{0}$, it follows from (W1'), (2.12), (2.20), (2.21) and (2.22) that

$$
\begin{aligned}
\sum_{n \in \mathbb{Z}}|W(n, u(n))| & \leq \sum_{i=1}^{2} a_{i} \sum_{n \in \mathbb{Z}}\left(1+|n|^{\sigma_{i}}\right)|u(n)|^{\gamma_{i}} \\
& \leq \sum_{i=1}^{2} a_{i}\left[\left(\sum_{|n| \leq N}\left(1+|n|^{\sigma_{i}}\right)^{2 /\left(2-\gamma_{i}\right)}[\tilde{l}(n)]^{-\gamma_{i} /\left(2-\gamma_{i}\right)}\right)^{1-\frac{\gamma_{i}}{2}}+\frac{K\left(\sigma_{i}, \gamma_{i}\right)}{N^{\kappa_{i}}}\right]\|u\|^{\gamma_{i}} \\
& =\phi_{1}(N)\|u\|^{\gamma_{1}}+\phi_{2}(N)\|u\|^{\gamma_{2}} .
\end{aligned}
$$

This shows that (2.19) holds.

Lemma 2.5 Assume that $L$ and $W$ satisfy $\left(\mathrm{L}_{v}^{\prime}\right),\left(\mathrm{W} 1^{\prime}\right)$ and (W2'). Then the functional $f$ : $E \rightarrow \mathbb{R}$ defined by

$$
f(u)=\frac{1}{2} b(u, u)-\sum_{n \in \mathbb{Z}} W(n, u(n)), \quad \forall u \in E
$$

is well defined and of class $C^{1}(E, \mathbb{R})$ and

$$
\left\langle f^{\prime}(u), v\right\rangle=b(u, v)-\sum_{n \in \mathbb{Z}}(\nabla W(n, u(n)), v(n)), \quad \forall u, v \in E .
$$

Furthermore, the critical points off in E are the solutions of system (1.1) with $u( \pm \infty)=0$. 
Proof Lemmas 2.2 and 2.4 imply that $f$ defined by (2.23) is well defined on $E$. Next, we prove that (2.24) holds. By (W2'), there exist $M_{1}, M_{2}>0$ such that

$$
\varphi_{i}(|x|) \leq M_{i}|x|^{\gamma_{2+i^{-1}}}, \quad \forall x \in \mathbb{R}^{\mathcal{N}},|x| \leq 1, i=1,2
$$

For any $u, v \in E$, there exists an integer $N_{1}>N_{0}$ such that $|u(n)|+|v(n)|<1$ for $|n|>N_{1}$. Then, for any sequence $\left\{\theta_{n}\right\}_{n \in \mathbb{Z}} \subset \mathbb{R}$ with $\left|\theta_{n}\right|<1$ for $n \in \mathbb{Z}$ and any number $h \in(0,1)$, by (W2'), (2.11) and (2.25), we have

$$
\begin{aligned}
& \sum_{n \in \mathbb{Z}} \max _{h \in[0,1]}\left|\left(\nabla W\left(n, u(n)+\theta_{n} h v(n)\right), v(n)\right)\right| \\
& \leq \sum_{|n| \leq N_{1}} \max _{h \in[0,1]}\left|\nabla W\left(n, u(n)+\theta_{n} h v(n)\right)\right||v(n)| \\
& +\sum_{|n|>N_{1}} \max _{h \in[0,1]}\left|\nabla W\left(n, u(n)+\theta_{n} h v(n)\right)\right||v(n)| \\
& \leq \sum_{|n| \leq N_{1}} \max _{|x| \leq\|u\|_{\infty}+\|v\|_{\infty}}|\nabla W(n, x) \| v(n)| \\
& +\sum_{i=1}^{2} M_{i} \sum_{|n|>N_{1}}\left(1+|n|^{\sigma_{i}}\right)(|u(n)|+|v(n)|)^{\gamma_{2+i}-1}|v(n)| \\
& \leq \sum_{|n| \leq N_{1}} \max _{|x| \leq\|u\|_{\infty}+\|v\|_{\infty}}|\nabla W(n, x)||v(n)|+\sum_{i=1}^{2} M_{i} \sum_{|n|>N_{1}}\left(1+|n|^{\sigma_{i}}\right)|v(n)|^{\gamma_{2+i}} \\
& +\sum_{i=1}^{2} M_{i}\left(\sum_{|n|>N_{1}}\left(1+|n|^{\sigma_{i}}\right)|u(n)|^{\gamma_{2+i}}\right)^{1-\frac{1}{\gamma_{2+i}}} \\
& \times\left(\sum_{|n|>N_{1}}\left(1+|n|^{\sigma_{i}}\right)|v(n)|^{\gamma_{2+i}}\right)^{\frac{1}{\gamma_{2+i}}} \\
& \leq \sum_{|n| \leq N_{1}} \max _{|x| \leq\|u\|_{\infty}+\|v\|_{\infty}}|\nabla W(n, x)||v(n)| \\
& +\sum_{i=1}^{2} \frac{M_{i} K\left(\sigma_{i}, \gamma_{2+i}\right)}{N_{1}^{\kappa_{2+i}}}\left(\|u\|^{\gamma_{2+i}-1}+\|v\|^{\gamma_{2+i}-1}\right)\|v\|<+\infty,
\end{aligned}
$$

where $\kappa_{2+i}=\left[\gamma_{2+i}(3-v)-2\left(1+\sigma_{i}\right)\right] / 2>0, i=1,2$. Then by (2.23), (2.26) and Lebesgue's dominated convergence theorem, we have

$$
\begin{aligned}
\left\langle f^{\prime}(u), v\right\rangle & =\lim _{h \rightarrow 0^{+}} \frac{f(u+h v)-f(u)}{h} \\
& =\lim _{h \rightarrow 0^{+}}\left[b(u, v)+\frac{h b(v, v)}{2}-\sum_{n \in \mathbb{Z}}\left(\nabla W\left(n, u(n)+\theta_{n} h v(n)\right), v(n)\right)\right] \\
& =b(u, v)-\sum_{n \in \mathbb{Z}}(\nabla W(n, u(n)), v(n)) .
\end{aligned}
$$

This shows that (2.24) holds. In view of the proof of [13, Lemma 2.6], the critical points of $f$ in $E$ are the solutions of system (1.1) with $u( \pm \infty)=0$. 
Let us prove now that $f^{\prime}$ is continuous. Let $u_{k} \rightarrow u$ in $E$. Then there exists a constant $\delta>0$ such that

$$
\|u\| \leq \sqrt[4]{\left(l^{*}+4 \alpha\right) l^{*}} \delta, \quad\left\|u_{k}\right\| \leq \sqrt[4]{\left(l^{*}+4 \alpha\right)} l^{*} \delta, \quad k=1,2, \ldots
$$

It follows from (2.6) that

$$
\|u\|_{\infty} \leq \delta, \quad\left\|u_{k}\right\|_{\infty} \leq \delta, \quad k=1,2, \ldots
$$

By (W2'), there exist $M_{3}, M_{4}>0$ such that

$$
\varphi_{i}(|x|) \leq M_{2+i}|x|^{\gamma_{2+i}-1}, \quad \forall x \in \mathbb{R}^{\mathcal{N}},|x| \leq \delta, i=1,2 .
$$

From (2.11), (2.24), (2.27), (2.28), (2.29), (W2') and the Hölder inequality, we have

$$
\begin{aligned}
& \left|\left\langle f^{\prime}\left(u_{k}\right)-f^{\prime}(u), v\right\rangle\right| \\
& \leq\left|b\left(u_{k}-u, v\right)\right|+\sum_{n \in \mathbb{Z}}\left|\left(\nabla W\left(n, u_{k}(n)\right)-\nabla W(n, u(n)), v(n)\right)\right| \\
& \leq C_{0}\left\|u_{k}-u\right\|\|v\|+\sum_{|n| \leq N}\left|\nabla W\left(n, u_{k}(n)\right)-\nabla W(n, u(n))\right||v(n)| \\
& \quad+\sum_{|n|>N}\left(\left|\nabla W\left(n, u_{k}(n)\right)\right|+|\nabla W(n, u(n))|\right)|v(n)| \\
& \leq o(1)+\sum_{i=1}^{2} M_{2+i} \sum_{|n|>N}\left(1+|n|^{\sigma_{i}}\right)\left(\left|u_{k}(n)\right|^{\gamma_{2+i}-1}+|u(n)|^{\gamma_{2+i}-1}\right)|v(n)| \\
& \leq o(1)+\sum_{i=1}^{2} M_{2+i}\left(\sum_{|n|>N}\left(1+|n|^{\sigma_{i}}\right)\left|u_{k}(n)\right|^{\gamma_{2+i}}\right)^{1-\frac{1}{\gamma_{2+i}}}\left(\sum_{|n|>N}\left(1+|n|^{\sigma_{i}}\right)|v(n)|^{\gamma_{2+i}}\right)^{\frac{1}{\gamma_{2+i}}} \\
& \quad+\sum_{i=1}^{2} M_{2+i}\left(\sum_{|n|>N}\left(1+|n|^{\sigma_{i}}\right)|u(n)|^{\gamma_{2+i}}\right)^{1-\frac{1}{\gamma_{2+i}}}\left(\sum_{|n|>N}\left(1+|n|^{\sigma_{i}}\right)|v(n)|^{\gamma_{2+i}}\right)^{\frac{1}{\gamma_{2+i}}} \\
& \leq o(1)+\sum_{i=1}^{2} \frac{M_{2+i} K\left(\sigma_{i}, \gamma_{2+i}\right)}{N^{\kappa_{2+i}}}\left(\left\|u_{k}\right\|^{\gamma_{2+i}-1}+\|u\|^{\gamma_{2+i}-1}\right)\|v\| \\
& =o(1), \quad k \rightarrow+\infty, N \rightarrow+\infty, \forall v \in E,
\end{aligned}
$$

which implies the continuity of $f^{\prime}$. The proof is complete.

Lemma 2.6 [14] Let $X$ be an infinite dimensional Banach space and let $f \in C^{1}(X, \mathbb{R})$ be even, satisfy the $(P S)$-condition, and $f(0)=0$. If $X=X_{1} \oplus X_{2}$ (direct sum), where $X_{1}$ is finite dimensional, and $f$ satisfies

(i) $f$ is bounded from below on $X_{2}$;

(ii) for each finite dimensional subspace $\tilde{X} \subset X$, there are positive constants $\rho=\rho(\tilde{X})$ and $\sigma=\sigma(\tilde{X})$ such that $\left.f\right|_{B_{\rho} \cap \tilde{X}} \leq 0$ and $\left.f\right|_{\partial B_{\rho} \cap \tilde{X}} \leq-\sigma$, where $B_{\rho}=\{x \in X:\|x\|=\rho\}$.

Then $f$ possesses infinitely many nontrivial critical points. 


\section{Proof of the theorem}

Proof of Theorem 1.2 For $u \in E$, we define two functions as follows:

$$
u^{-}(n)=\left\{\begin{array}{ll}
u(n), & n \in \mathbb{Z}^{1}, \\
0, & n \in \mathbb{Z}^{2} ;
\end{array} \quad u^{+}(n)= \begin{cases}0, & n \in \mathbb{Z}^{1}, \\
u(n), & n \in \mathbb{Z}^{2} .\end{cases}\right.
$$

Set

$$
X_{1}=\left\{u^{-}: u \in E\right\}, \quad X_{2}=\left\{u^{+}: u \in E\right\} .
$$

Then $X:=E=X_{1} \oplus X_{2}$ (direct sum) and $\operatorname{dim}\left(X_{1}\right)<+\infty$. Obviously, (W1') and (W5) imply $f(0)=0$ and $f$ is even. In view of Lemma $2.5, f \in C^{1}(E, \mathbb{R})$. In what follows, we first prove that $f$ satisfies the (PS)-condition. Assume that $\left\{u_{k}\right\}_{k \in \mathbb{N}} \subset E$ is a (PS)-sequence: $\left\{f\left(u_{k}\right)\right\}_{k \in \mathbb{N}}$ is bounded and $\left\|f^{\prime}\left(u_{k}\right)\right\| \rightarrow 0$ as $k \rightarrow+\infty$. From (2.23), (2.24) and (W3'), we have

$$
\begin{aligned}
\left\langle f^{\prime}\left(u_{k}\right), u_{k}\right\rangle-2 f\left(u_{k}\right) & =\sum_{n \in \mathbb{Z}}\left[2 W\left(n, u_{k}(n)\right)-\left(\nabla W\left(n, u_{k}(n)\right), u_{k}(n)\right)\right] \\
& \geq b_{1} \sum_{n \in \mathbb{Z}}\left|u_{k}(n)\right|^{\gamma_{5}}-b_{2} \sum_{n \in \mathbb{Z}}\left|u_{k}(n)\right|^{\gamma_{6}} \\
& =b_{1}\left\|u_{k}\right\|_{\gamma_{5}}^{\gamma_{5}}-b_{2}\left\|u_{k}\right\|_{\gamma_{6}}^{\gamma_{6}} .
\end{aligned}
$$

It follows that there exists a constant $C_{1}>0$ such that

$$
b_{1}\left\|u_{k}\right\|_{\gamma_{5}}^{\gamma_{5}}-b_{2}\left\|u_{k}\right\|_{\gamma_{6}}^{\gamma_{6}} \leq C_{1}\left(1+\left\|u_{k}\right\|\right)
$$

Since $\operatorname{dim}\left(X_{1}\right)<+\infty$, it follows that there exists a constant $C_{2}>0$ such that

$$
\left\|u_{k}^{-}\right\|_{2}^{2}=\left(u_{k}^{-}, u_{k}\right)_{l^{2}} \leq\left\|u_{k}^{-}\right\|_{\gamma_{5}^{\prime}}\left\|u_{k}\right\|_{\gamma_{5}} \leq C_{2}\left\|u_{k}^{-}\right\|_{2}\left\|u_{k}\right\|_{\gamma_{5}}
$$

where $\gamma_{5}^{\prime}=\gamma_{5} /\left(\gamma_{5}-1\right)$. Combining (3.3) with (3.4), one has

$$
\begin{aligned}
\sum_{n \in \mathbb{Z}^{1}}\left((\tilde{L}(n)-L(n)) u_{k}(n), u_{k}(n)\right) & =\sum_{n \in \mathbb{Z}^{1}}\left((\tilde{L}(n)-L(n)) u_{k}^{-}(n), u_{k}^{-}(n)\right) \\
& \leq C_{3}\left\|u_{k}^{-}\right\|_{2}^{2} \\
& \leq C_{4}\left(1+\left\|u_{k}\right\|^{2 / \gamma_{5}}+\left\|u_{k}\right\|^{2 \gamma_{6} / \gamma_{5}}\right) .
\end{aligned}
$$

From (2.19), (2.23) and (3.5), we obtain

$$
\begin{aligned}
\left\|u_{k}\right\|^{2} & =\sum_{n \in \mathbb{Z}}\left[\left(p(n+1) \Delta u_{k}(n), \triangle u_{k}(n)\right)+\left(\tilde{L}(n) u_{k}(n), u_{k}(n)\right)\right] \\
& =b\left(u_{k}, u_{k}\right)+\sum_{n \in \mathbb{Z}^{1}}\left((\tilde{L}(n)-L(n)) u_{k}(n), u_{k}(n)\right) \\
& =\sum_{n \in \mathbb{Z}^{1}}\left((\tilde{L}(n)-L(n)) u_{k}(n), u_{k}(n)\right)+2 f\left(u_{k}\right)+2 \sum_{n \in \mathbb{Z}} W\left(n, u_{k}(n)\right)
\end{aligned}
$$




$$
\begin{aligned}
\leq & C_{5}\left(1+\left\|u_{k}\right\|^{2 / \gamma_{5}}+\left\|u_{k}\right\|^{2 \gamma_{6} / \gamma_{5}}\right) \\
& +2 \phi_{1}\left(N_{0}\right)\left\|u_{k}\right\|^{\gamma_{1}}+2 \phi_{2}\left(N_{0}\right)\left\|u_{k}\right\|^{\gamma_{2}} \\
\leq & C_{6}\left(1+\left\|u_{k}\right\|^{\gamma_{1}}+\left\|u_{k}\right\|^{\gamma_{2}}+\left\|u_{k}\right\|^{2 / \gamma_{5}}+\left\|u_{k}\right\|^{2 \gamma_{6} / \gamma_{5}}\right) .
\end{aligned}
$$

Since $1<\gamma_{1}<\gamma_{2}<2,1<\gamma_{6}<\gamma_{5}<2$, it follows from (3.6) that $\left\{\left\|u_{k}\right\|\right\}$ is bounded. Let $A>0$ such that

$$
\left\|u_{k}\right\|_{\infty} \leq \frac{1}{\sqrt[4]{\left(l_{*}+4 \alpha\right) l_{*}}}\left\|u_{k}\right\| \leq A, \quad k \in \mathbb{N} .
$$

So, passing to a subsequence if necessary, it can be assumed that $u_{k} \rightarrow u_{0}$ in $E$. It is easy to verify that

$$
\lim _{k \rightarrow \infty} u_{k}(n)=u_{0}(n), \quad \forall n \in \mathbb{Z}
$$

By (W2'), there exist $M_{5}, M_{6}>0$ such that

$$
\varphi_{i}(|x|) \leq M_{4+i}|x|^{\gamma_{2+i}-1}, \quad \forall x \in \mathbb{R}^{\mathcal{N}},|x| \leq A, i=1,2 .
$$

For any given number $\varepsilon>0$, we can choose an integer $N_{3}>N_{0}$ such that

$$
\frac{M_{4+i} K\left(\sigma_{i}, \gamma_{2+i}\right)}{N_{3}^{\kappa_{2+i}}}\left\{\left[\sqrt[4]{\left(l_{*}+4 \alpha\right) l_{*}} A\right]^{\gamma_{2+i}}+\left\|u_{0}\right\|^{\gamma_{2+i}}\right\}<\varepsilon, \quad i=1,2 .
$$

It follows from (3.8) and the continuity of $\nabla W(n, x)$ on $x$ that there exists $k_{0} \in \mathbb{N}$ such that

$$
\sum_{n=-N_{2}}^{N_{2}}\left|\nabla W\left(n, u_{k}(n)\right)-\nabla W\left(n, u_{0}(n)\right)\right|\left|u_{k}(n)-u_{0}(n)\right|<\varepsilon \quad \text { for } k \geq k_{0}
$$

On the other hand, it follows from (2.11), (3.7), (3.9), (3.10) and (W2') that

$$
\begin{aligned}
& \sum_{|n|>N_{2}}\left|\nabla W\left(n, u_{k}(n)\right)-\nabla W\left(n, u_{0}(n)\right)\right|\left|u_{k}(n)-u_{0}(n)\right| \\
& \leq \sum_{|n|>N_{2}}\left[\left|\nabla W\left(n, u_{k}(n)\right)\right|+\left|\nabla W\left(n, u_{0}(n)\right)\right|\right]\left(\left|u_{k}(n)\right|+\left|u_{0}(n)\right|\right) \\
& \leq \sum_{i=1}^{2} \sum_{|n|>N_{2}}\left(1+|n|^{\sigma_{i}}\right)\left[\varphi_{i}\left(\left|u_{k}(n)\right|\right)+\varphi_{i}\left(\left|u_{0}(n)\right|\right)\right]\left(\left|u_{k}(n)\right|+\left|u_{0}(n)\right|\right) \\
& \leq \sum_{i=1}^{2} M_{4+i} \sum_{|n|>N_{2}}\left(1+|n|^{\sigma_{i}}\right)\left(\left|u_{k}(n)\right|^{\gamma_{2+i}-1}+\left|u_{0}(n)\right|^{\gamma_{2+i}-1}\right)\left(\left|u_{k}(n)\right|+\left|u_{0}(n)\right|\right) \\
& \leq 2 \sum_{i=1}^{2} M_{4+i} \sum_{|n|>N_{2}}\left(1+|n|^{\sigma_{i}}\right)\left(\left|u_{k}(n)\right|^{\gamma_{2+i}}+\left|u_{0}(n)\right|^{\gamma_{2+i}}\right) \\
& \leq \sum_{i=1}^{2} \frac{2 M_{4+i} K\left(\sigma_{i}, \gamma_{2+i}\right)}{N_{2}^{\kappa_{2+i}}}\left(\left\|u_{k}\right\|^{\gamma_{2+i}}+\left\|u_{0}\right\|^{\gamma_{2+i}}\right)
\end{aligned}
$$




$$
\begin{aligned}
& \leq \sum_{i=1}^{2} \frac{2 M_{4+i} K\left(\sigma_{i}, \gamma_{2+i}\right)}{N_{2}^{\kappa_{2+i}}}\left\{\left[\sqrt[4]{\left(l_{*}+4 \alpha\right) l_{*}} A\right]^{\gamma_{2+i}}+\left\|u_{0}\right\|^{\gamma_{2+i}}\right\} \\
& \leq 4 \varepsilon, \quad k \in \mathbb{N} .
\end{aligned}
$$

Since $\varepsilon$ is arbitrary, combining (3.11) with (3.12), we get

$$
\sum_{n \in \mathbb{Z}}\left(\nabla W\left(n, u_{k}(n)\right)-\nabla W\left(n, u_{0}(n)\right), u_{k}(n)-u_{0}(n)\right) \rightarrow 0 \quad \text { as } k \rightarrow \infty .
$$

It follows from (2.24) that

$$
\begin{aligned}
& \left\langle f^{\prime}\left(u_{k}\right)-f^{\prime}\left(u_{0}\right), u_{k}-u_{0}\right\rangle \\
& =b\left(u_{k}-u_{0}, u_{k}-u_{0}\right)-\sum_{n \in \mathbb{Z}}\left(\nabla W\left(n, u_{k}(n)\right)-\nabla W\left(n, u_{0}(n)\right), u_{k}(n)-u_{0}(n)\right) \\
& =\left\|u_{k}-u_{0}\right\|^{2}-\sum_{n \in \mathbb{Z}^{1}}\left((\tilde{L}(n)-L(n))\left(u_{k}-u_{0}\right), u_{k}-u_{0}\right) \\
& \quad-\sum_{n \in \mathbb{Z}}\left(\nabla W\left(n, u_{k}(n)\right)-\nabla W\left(n, u_{0}(n)\right), u_{k}(n)-u_{0}(n)\right) .
\end{aligned}
$$

Since $\left\langle f^{\prime}\left(u_{k}\right)-f^{\prime}\left(u_{0}\right), u_{k}-u_{0}\right\rangle \rightarrow 0$, it follows from (3.8), (3.13) and (3.14) that $u_{k} \rightarrow u_{0}$ in $E$. Hence, $f$ satisfies the (PS)-condition.

Next, for $u \in X_{2}$, it follows from (2.9), (2.19) and (2.23) that

$$
\begin{aligned}
f(u) & =\frac{1}{2} b(u, u)-\sum_{n \in \mathbb{Z}} W(n, u(n))=\frac{1}{2}\|u\|^{2}-\sum_{n \in \mathbb{Z}} W(n, u(n)) \\
& \geq \frac{1}{2}\|u\|^{2}-\phi_{1}\left(N_{0}\right)\|u\|^{\gamma_{1}}-\phi_{2}\left(N_{0}\right)\|u\|^{\gamma_{2}} \rightarrow+\infty
\end{aligned}
$$

as $\|u\| \rightarrow+\infty$ and $u \in X_{2}$, since $1<\gamma_{1}<\gamma_{2}<2$.

Finally, we prove that assumption (ii) in Lemma 2.6 holds. Let $\tilde{X} \subset X$ be any finite dimensional subspace. Then there exist constants $c_{0}=c(\tilde{X})>0$ and $c_{*}=c(\tilde{X})>0$ such that

$$
c_{0}\|u\| \leq\|u\|_{\gamma_{i}} \leq c_{*}\|u\|, \quad \forall i=7,8, u \in \tilde{X}
$$

From (2.9), (2.23), (3.16) and (W4'), one has

$$
\begin{aligned}
f(u) & =\frac{1}{2} b(u, u)-\sum_{n \in \mathbb{Z}} W(n, u(n)) \\
& \leq \frac{1}{2}\|u\|^{2}-b_{3} \sum_{n \in \mathbb{Z}}|u(n)|^{\gamma_{7}}+b_{4} \sum_{n \in \mathbb{Z}}|u(n)|^{\gamma_{8}} \\
& =\frac{1}{2}\|u\|^{2}-b_{3}\|u\|_{\gamma_{7}}^{\gamma_{7}}+b_{4}\|u\|_{\gamma_{8}}^{\gamma_{8}} \\
& \leq \frac{1}{2}\|u\|^{2}-b_{3} c_{0}^{\gamma_{7}}\|u\|^{\gamma_{7}}+b_{4} c_{*}^{\gamma_{8}}\|u\|^{\gamma_{8}}, \quad \forall u \in \tilde{X} .
\end{aligned}
$$


Since $1<\gamma_{7}<\gamma_{8}<2$, the above estimation implies that there exist $\rho=\rho\left(b_{3}, b_{4}, c_{0}, c_{*}\right)=$ $\rho(\tilde{X})>0$ and $\sigma=\sigma\left(b_{3}, b_{4}, c_{0}, c_{*}\right)=\sigma(\tilde{X})>0$ such that

$$
f(u) \leq 0, \quad \forall u \in B_{\rho} \cap \tilde{X} ; \quad f(u) \leq-\sigma, \quad \forall u \in \partial B_{\rho} \cap \tilde{X} .
$$

This shows that assumption (ii) in Lemma 2.6 holds. By Lemma 2.6, $f$ has infinitely many critical points which are homoclinic solutions for system (1.1).

\section{Example}

In this section, we give an example to illustrate our result.

Example 4.1 In system (1.1), let $p(n)$ be an $\mathcal{N} \times \mathcal{N}$ real symmetric positive definite matrix for all $n \in \mathbb{Z}, L(n)=\left(1+\sin ^{2} n\right)\left(|n|^{4 / 5}-6\right) I_{\mathcal{N}}$, and let

$$
W(n, x)=\left(1+\sin ^{2} n\right)\left[\left(1+|n|^{1 / 9}\right)|x|^{5 / 4}-3|x|^{3 / 2}+\left(1+|n|^{1 / 2}\right)|x|^{7 / 4}\right] .
$$

Then $L$ satisfies $\left(L_{v}^{\prime}\right)$ with $v=6 / 5$, and

$$
\begin{aligned}
& \nabla W(n, x)=\left(1+\sin ^{2} n\right)\left[\frac{5}{4}\left(1+|n|^{1 / 9}\right)|x|^{-3 / 4} x-\frac{9}{2}|x|^{-1 / 2} x+\frac{7}{4}\left(1+|n|^{1 / 2}\right)|x|^{-1 / 4} x\right], \\
& |W(n, x)| \leq 5\left(1+|n|^{1 / 9}\right)|x|^{5 / 4}+5\left(1+|n|^{1 / 2}\right)|x|^{7 / 4}, \quad \forall(n, x) \in \mathbb{Z} \times \mathbb{R}^{N}, \\
& |\nabla W(n, x)| \leq 7\left(1+|n|^{1 / 9}\right)|x|^{1 / 4}+8\left(1+|n|^{1 / 2}\right)|x|^{3 / 4}, \quad \forall(n, x) \in \mathbb{Z} \times \mathbb{R}^{N}, \\
& 2 W(n, x)-\nabla W(n, x) \geq \frac{1}{4}|x|^{7 / 4}-3|x|^{3 / 2}, \quad \forall(n, x) \in \mathbb{Z} \times \mathbb{R}^{N}
\end{aligned}
$$

and

$$
W(n, x) \geq|x|^{5 / 4}-6|x|^{3 / 2}, \quad \forall(n, x) \in \mathbb{Z} \times \mathbb{R}^{N} .
$$

Thus all the conditions of Theorem 1.2 are satisfied with

$$
\begin{aligned}
& \frac{5}{4}=\gamma_{1}=\gamma_{3}=\gamma_{7}<\gamma_{6}=\gamma_{8}=\frac{3}{2}<\gamma_{5}=\gamma_{4}=\gamma_{2}=\frac{7}{4} ; \\
& a_{1}=a_{2}=5 ; \quad b_{1}=\frac{1}{4}, \quad b_{2}=3, \quad b_{3}=1, \quad b_{4}=6 ; \\
& \sigma_{1}=\frac{1}{9}, \quad \sigma_{2}=\frac{1}{2} ; \quad \varphi_{1}(s)=7 s^{1 / 4}, \quad \varphi_{2}(s)=8 s^{3 / 4} .
\end{aligned}
$$

Hence, by Theorem 1.2, system (1.1) has infinitely many nontrivial homoclinic solutions. However, one can see that $W(n, x)$ defined by (4.1) does not satisfy (W1) and (W2).

Competing interests

The author declares that they have no competing interests.

\section{Acknowledgements}

The author would like to express their thanks to the referees for their helpful suggestions. This work is partially supported by the NNSF (No. 11171351) of China and supported by the Scientific Research Fund of Hunan Provincial Education Department (08A053) and supported by the Hunan Provincial Natural Science Foundation of China (No. 11 JJ2005). 
References

1. Agarwal, RP: Difference Equations and Inequalities: Theory, Methods and Applications, 2nd edn. Dekker, New York (2000)

2. Ahlbrandt, CD, Peterson, AC: Discrete Hamiltonian Systems: Difference Equations, Continued Fraction and Riccati Equations. Kluwer Academic, Dordrecht (1996)

3. Chen, $\mathrm{P}$, Tang, $\mathrm{XH}$, Agarwal, RP: Homoclinic solutions for second order differential equations generated by impulses. Adv. Math. Sci. Appl. 21, 447-465 (2011)

4. Deng, $X Q$, Cheng, G: Homoclinic orbits for second order discrete Hamiltonian systems with potential changing sign. Acta Appl. Math. 103, 301-314 (2008). doi:10.1007/s10440-008-9237-z

5. Guo, C, O'Regan, D, Xu, Y, Agarwal, RP: Existence and multiplicity of homoclinic orbits of a second-order differential difference equation via variational methods. Sci. Publ. State Univ. Novi Pazar Ser. A, Appl. Math. Inf. Mech. 4, 1-15 (2012)

6. Guo, C, O'Regan, D, Xu, Y, Agarwal, RP: Homoclinic orbits for a singular second-order neutral differential equation. J. Math. Anal. Appl. 366, 550-560 (2010)

7. Ma, M, Guo, ZM: Homoclinic orbits for second order self-adjoint difference equations. J. Math. Anal. Appl. 323(1), 513-521 (2006)

8. Ma, M, Guo, ZM: Homoclinic orbits and subharmonics for nonlinear second order difference equations. Nonlinear Anal. 67, 1737-1745 (2007)

9. Lin, $X Y$, Tang, $X H$ : Existence of infinitely many homoclinic orbits in discrete Hamiltonian systems. J. Math. Anal. Appl. 373, 59-72 (2011)

10. Lin, $X Y$, Tang, $X H$ : Homoclinic orbits for discrete Hamiltonian systems with subquadratic potential (to appear)

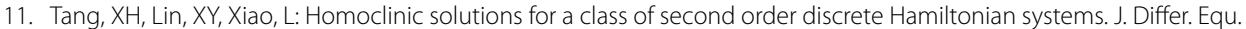
Appl. 16, 1257-1273 (2010)

12. Tang, $\mathrm{XH}$, Lin, $\mathrm{XY}$ : Existence and multiplicity of homoclinic solutions for second-order discrete Hamiltonian systems with subquadratic potential. J. Differ. Equ. Appl. 17, 1617-1634 (2011)

13. Tang, $\mathrm{XH}$, Lin, $\mathrm{XY}$ : Infinitely many homoclinic orbits for discrete Hamiltonian systems with subquadratic potential. J. Differ. Equ. Appl. 19, 796-813 (2013)

14. Ding, YH: Existence and multiplicity results for homoclinic solutions to a class of Hamiltonian systems. Nonlinear Anal. 25(11), 1095-1113 (1995)

doi:10.1186/1687-1847-2013-228

Cite this article as: Lin: Homoclinic orbits for second-order discrete Hamiltonian systems with subquadratic

potential. Advances in Difference Equations 2013 2013:228.

\section{Submit your manuscript to a SpringerOpen ${ }^{\circ}$ journal and benefit from:}

- Convenient online submission

Rigorous peer review

- Immediate publication on acceptance

- Open access: articles freely available online

- High visibility within the field

- Retaining the copyright to your article

Submit your next manuscript at $>$ springeropen.com 